ONCOGENES

\section{Receptor relay}

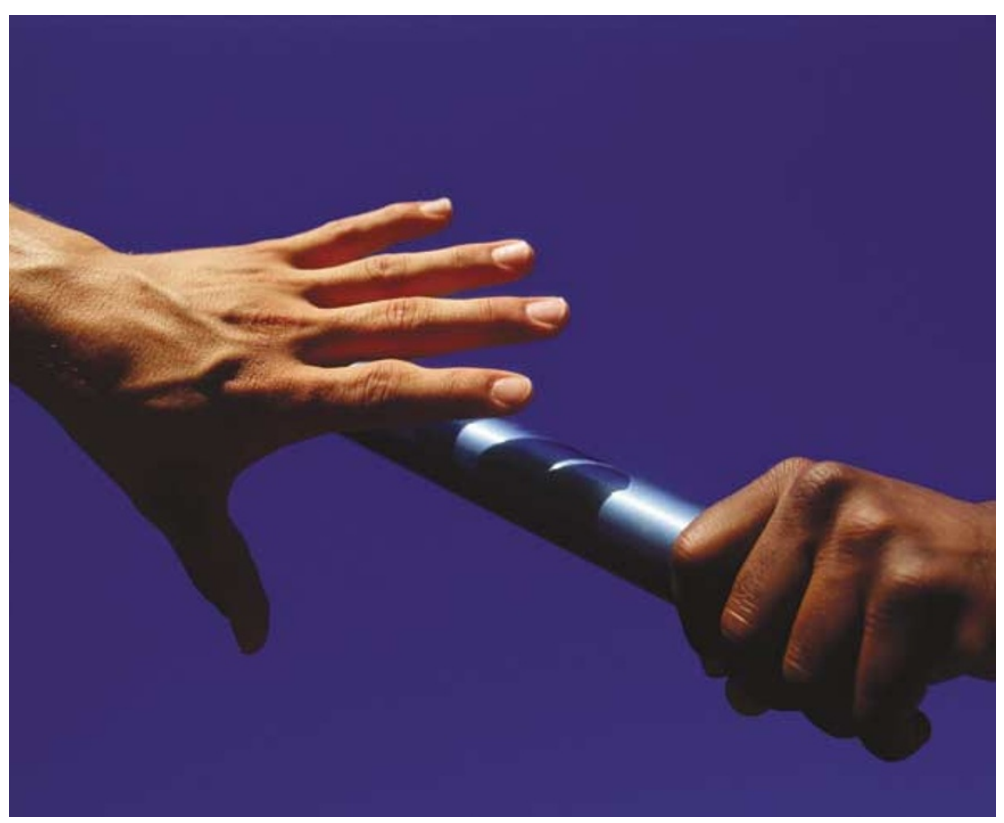

c-SRC is a common oncogenic partner of the epidermal growth-factor receptor (EGFR), as both c-SRC and EGFR are overexpressed in human malignancies such as breast cancer. However, the mode of oncogenic cooperation between these two proteins has been unclear. Now, Yosef Yarden and colleagues report that c-CBL - a ubiquitin ligase that is known to be a regulator of EGFR endocytosis - is the 'middle leg' in the relay of oncogenic signalling between c-SRC and EGFR.

To investigate whether c-SRC affects the expression of EGFR, the receptor was co-expressed with different forms of c-SRC in receptornegative Chinese hamster ovary (CHO) cells. Both wild-type c-SRC and an active-mutant form resulted in increased levels of expression of EGFR, in contrast to a kinase-defective mutant, which led to decreased EGFR expression levels. As the active form of c-SRC was shown to have no effect on receptor synthesis (mRNA levels) or maturation, the authors propose that c-SRC stabilizes the mature, cell-surface form of EGFR.

c-CBL ubiquitylates EGFR, which results in its endocytosis and degradation and, therefore, receptor desensitization in response to growth-factor signalling. Could effects on c-CBL be responsible for the change in expression of EGFR in response to c-SRC? Ectopic expression of $\mathrm{c}-\mathrm{CBL}$ increased the removal of EGFR from the surface of SYF cells (which lack SRC, YES and FYN), but this receptor endocytosis was inhibited by the co-expression of an active-mutant c-SRC. The authors found that c-SRC prevents the c-CBL-EGFR interaction (which is required for receptor ubiquitylation), specifically downregulates the expression of c-CBL and recruits c-CBL to vesicles.

So, how does c-SRC decrease the level of expression of c-CBL? Expression of active-mutant c-SRC leads to polyubiquitylation of c-CBL, which targets this protein for degradation by the proteasome. This process does not occur for a RING-finger

\title{
Back in time
}

A number of developmental signalling pathways have been shown to be reactivated during tumour formation - the Hedgehog (HH) pathway seems to be the latest member of this growing list. $\mathrm{HH}$ signalling mediates pattern formation during embryogenesis, and has recently been shown to regulate epithelial-mesenchymal interactions during lung development. In Nature, Watkins et al. now report that $\mathrm{HH}$ signalling also promotes lung tumour development.

Sonic Hedgehog $(\mathrm{SHH})$ - a secreted ligand for the $\mathrm{HH}$ receptor patched (PTCH) - is a signalling switch expressed by a variety of differentiation subpopulations of cells throughout the embryo. Loss of Shh function results in severe lung defects in mice. Unlike skin and colon, the adult airway epithelium only proliferates in response to injury. In a search for factors that activate airway epithelial-cell proliferation after injury, Watkins et al. observed increased expression of both Shh and its transcriptional effector Glil in an adult mouse model of acute airway repair.
This was surprising, as this pathway had been previously only associated with embryonic lung epithelial cells, where it signals adjacent lung mesenchyme to regulate branching morphogenesis. Watkins et al. next looked to see if $\mathrm{SHH}$ was upregulated in lung tumours. They examined different tumour types, and found that 5 of 10 human small-cell lung carcinoma (SCLC) samples expressed SHH and GLI1. Only 9 of 40 non-SCLC (NSCLC) tumour samples expressed $\mathrm{SHH}$, however, and only 4 of these also expressed GLI1. These findings indicate that the $\mathrm{HH}$ signalling pathway is reactivated in lung cancer cells - predominantly in SCLC.

But is ligand-driven $\mathrm{HH}$ pathway activation required for SCLC formation? Antibody inhibition of SHH prevented the growth of cultured SCLC cells. Furthermore, treatment of nine SCLC cell lines that expressed both SHH and GLI1 with cyclopamine - an alkaloid inhibitor of the HH pathway — induced both growth arrest and apoptosis. Cyclopamine had no effect on growth of NSCLC cells, and a closely related compound that does not inhibit $\mathrm{HH}$ signalling had no effect on SCLC cells. Cyclopamine also inhibited growth of three different SHH- and GLI1-expressing SCLC xenografts in nude mice, but not of NSCLC or colon cancer xenografts.

Activation of $\mathrm{HH}$ signalling has been previously associated with medulloblastoma. The HH pathway regulates cerebellar progenitor differentiation, and in this brain tumour it is believed to allow malignant cells to maintain progenitor-like fates. Similarly, SCLC might represent a malignancy that arises from an airway epithelial progenitor and has maintained its $\mathrm{HH}$ signalling capabilities, as these cells continue to express $\mathrm{SHH}$ and lack $\mathrm{PTCH}$ mutations. Drugs designed to inhibit $\mathrm{HH}$ signalling could therefore have therapeutic effects in patients with SCLC.

Kristine Novak

(1) References and links

ORIGINAL RESEARCH PAPER Watkins, D. N. et al

Hedgehog signalling within airway epithelial progenitors and small cell lung cancer. Nature 5 Mar 2003

(doi:10.1038.nature01493)

\section{FURTHER READING}

Berman, D. M. et al. Medulloblastoma growth inhibition by

Hedgehog pathway blockade. Science 297, 1559-1561 (2002) WEB SITE

Stephen Baylin's lab:

http://www.hopkinsmedicine.org/graduateprograms/cmm/b aylin.html 